\title{
Posterior tibial slope: the fingerprint of the tibial bone
}

\author{
Philipp W. Winkler ${ }^{1,2} \cdot$ Brian M. Godshaw ${ }^{1} \cdot$ Jon Karlsson $^{3} \cdot$ Alan M. J. Getgood $^{4} \cdot$ Volker Musahl $^{1}$
}

Received: 9 April 2021 / Accepted: 12 April 2021 / Published online: 27 April 2021

(c) European Society of Sports Traumatology, Knee Surgery, Arthroscopy (ESSKA) 2021

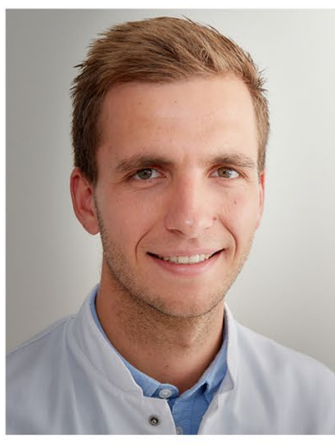

Philipp W. Winkler

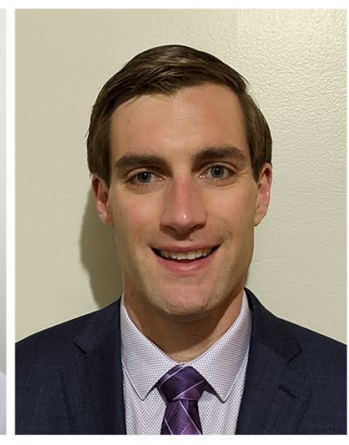

Brian M. Godshaw

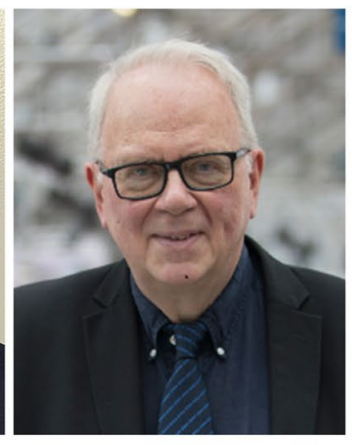

Jon Karlsson

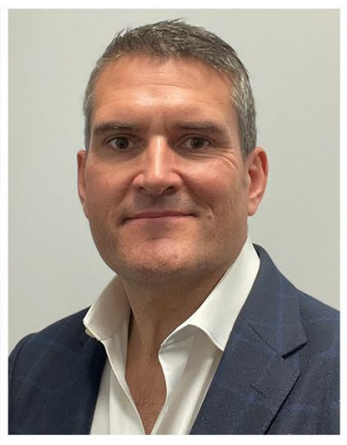

Alan M. J. Getgood

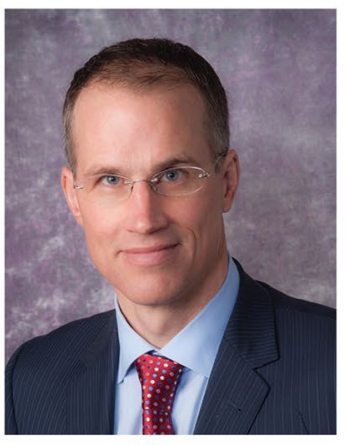

Volker Musahl
"Life is most delightful on the downward slope."

-Lucius Annaeus Seneca the Younger

It is not only downhill bikers and alpine skiers who show great interest in a steep and sometimes slippery slope. No, in recent years also orthopaedic surgeons eagerly pursue the posterior tibial slope (PTS), seeking answers of its biomechanical and clinical impact in knee function.

Why is there so much hype about the so-called "tibial slope"? The PTS represents the posterior inclination of the proximal tibia, making the PTS a three-dimensional,

Volker Musahl

musahlv@upmc.edu

1 Department of Orthopaedic Surgery, UPMC Freddie Fu Sports Medicine Center, University of Pittsburgh, 3200 S. Water St., Pittsburgh, PA 15203, USA

2 Department for Orthopaedic Sports Medicine, Klinikum Rechts Der Isar, Technical University of Munich, Ismaninger Str. 22, 81675 Munich, Germany

3 Department for Orthopaedics, Sahlgrenska University Hospital, Institute of Clinical Sciences, Sahlgrenska Academy, Gothenburg University, Gothenburg, Sweden

4 Department of Orthopedic Surgery, Fowler Kennedy Sport Medicine Clinic, University of Western Ontario, 3M Centre, 1151 Richmond Street, London, ON N6A 3K7, Canada individual characteristic of the tibia. Given significant PTS differences amongst sexes (female $>$ male), races (African $>$ Caucasian), and even compartments (medial $>$ lateral), the PTS may be referred to as the fingerprint of the tibial bone [13].

In 2004, two independent research facilities, one from the United States [5] and one from Germany [1], published landmark research on the effect of PTS-changing osteotomy on knee kinematics and contact mechanics. Consistently, it has been shown that an increase in PTS results in an anterior shift of the tibial resting position [1, 5]. Accordingly, it has been suggested that decreasing the PTS may improve anterior-posterior knee laxity in the anterior cruciate ligament (ACL) deficient knee [5]. The ingrowth of biomechanical knowledge of sagittal lower limb alignment gained from these landmark studies resulted in a surge of observational clinical studies focusing on PTS and ACL injury. It has been shown that high (steep) PTS is associated with an increased risk of primary and recurrent ACL injury [6, 9, 10, 14, 16]. Most remarkably, the association between high PTS and ACL injury was demonstrated in a study with the subtitle "The Catastrophic Effect of Age and Posterior Tibial Slope” [9]. The ACL graft failure rate was found to be 11-fold higher in adolescents ( $\leq 18$ years old at time of ACL reconstruction) with a PTS $\geq 12^{\circ}$ when compared to adults undergoing ACL reconstruction with 
PTS $<12^{\circ}$ [9]. The significantly higher ACL graft failure rate in adolescents with increased PTS demonstrates the catastrophic effect of PTS and age on ACL reconstruction.

Of course, every ACL surgeon intends to minimise the risk of ACL graft failure, particularly in young athletic patients. However, shall we really take action to modify the individual tibial fingerprint in patients with ACL injury? Or are we better off counselling patients regarding their increased risk of failure, look at different graft choices or potentially add in a lateral augmentation procedure? Or maybe smarter still, discuss a change in type, volume and intensity of sport participation? In the primary setting, these latter options may be a safer and more palapable offering, with the more aggressive osteotomy left for the revision or re-revision procedures. However, if we take a close look at the results from biomechanical labs around the world, there is no doubt that PTS reduction could be a reasonable consideration. Several well-designed biomechanical studies have found lower ACL graft forces after decreasing PTS $[3,7,8,15]$. Following the biomechanical evidence, clinicians now perform anterior closing wedge high tibial osteotomy to combine ACL reconstruction with PTS reduction. Since 2014, a total of four studies investigating primary (one study) and revision (three studies) ACL reconstruction combined with PTS reduction high tibial osteotomy have been published $[2,4,11,12]$. Satisfactory outcomes have consistently been reported in regards to postoperative anterior-posterior and rotatory knee laxity, patient-reported outcomes, and failure rates, after a minimum 2-year follow-up [2, 4, 11, 12]. However, it should be considered that the existing data are based on case series (i.e. no control group) with a total of 52 patients. In addition, long-term results have yet to be reported.

With this recent expansion of knowledge, PTS has worked its way to the forefront of ACL injury treatment. As orthopaedic surgeons, osteotomies are in our DNA; however, ideal indications for PTS reducing osteotomies still require refinement. The available evidence has taught us that anterior closing wedge high tibial osteotomy may lead to hyperextension [15]. This should be considered preoperatively, as iatrogenic creation of the extremely debilitating symptom of a hyperextension thrust must be avoided. The effect of PTS-changing osteotomy on native intra-articular structures such as the menisci or the posterior cruciate ligament are also largely unknown. Therefore, at the University of Pittsburgh, we felt compelled to investigate the impact of PTS change on these native intra-articular structures of the knee. In the future, this may help in selecting the ideal patient for PTS-changing osteotomy.

The incorporation of corrective coronal plane osteotomies into daily clinical practice amongst the orthopaedic community has taken not only excellent biomechanical and clinical research, but more importantly, years of experience.
Major efforts have succeeded and turned coronal plane corrective osteotomies into reliable and highly effective procedures that are now performed on a routine basis. Similarly, ongoing research efforts are devoted to determine precise indications for PTS corrective osteotomy. These efforts seek to present high-level evidence to introduce PTS corrective osteotomies to the daily clinical practice of every dedicated knee surgeon.

In conclusion, we recommend to assess the PTS in every patient with ACL injury. This information is extremely useful when counselling young patients as to their risk of re-injury and choice of surgical procedure. In second or multiple revision ACL reconstruction cases and when PTS is $>12^{\circ}$, PTS-decreasing high tibial osteotomies utilising supra-, trans-, or infra-tuberosity approaches could be considered to improve graft survivorship in the short term. We wait to see what advantages this may convey in the long term.

\section{References}

1. Agneskirchner JD, Hurschler C, Stukenborg-Colsman C, Imhoff AB, Lobenhoffer P (2004) Effect of high tibial flexion osteotomy on cartilage pressure and joint kinematics: a biomechanical study in human cadaveric knees. Winner of the AGA-DonJoy Award 2004. Arch Orthop Trauma Surg 124:575-584

2. Akoto R, Alm L, Drenck TC, Frings J, Krause M, Frosch KH (2020) Slope-correction osteotomy with lateral extra-articular tenodesis and revision anterior cruciate ligament reconstruction is highly effective in treating high-grade anterior knee laxity. Am J Sports Med. https://doi.org/10.1177/03635465209663273635 46520966327

3. Bernhardson AS, Aman ZS, Dornan GJ, Kemler BR, Storaci HW, Brady AW et al (2019) Tibial slope and its effect on force in anterior cruciate ligament grafts: anterior cruciate ligament force increases linearly as posterior tibial slope increases. Am J Sports Med 47:296-302

4. Dejour D, Saffarini M, Demey G, Baverel L (2015) Tibial slope correction combined with second revision ACL produces good knee stability and prevents graft rupture. Knee Surg Sports TraumatolArthrosc 23:2846-2852

5. Giffin JR, Vogrin TM, Zantop T, Woo SL, Harner CD (2004) Effects of increasing tibial slope on the biomechanics of the knee. Am J Sports Med 32:376-382

6. Grassi A, Macchiarola L, Urrizola Barrientos F, Zicaro JP, Costa Paz M, Adravanti P et al (2019) Steep posterior tibial slope, anterior tibial subluxation, deep posterior lateral femoral condyle, and meniscal deficiency are common findings in multiple anterior cruciate ligament failures: an MRI case-control study. Am J Sports Med 47:285-295

7. Imhoff FB, Comer B, Obopilwe E, Beitzel K, Arciero RA, Mehl JT (2020) Effect of slope and varus correction high tibial osteotomy in the ACL-deficient and ACL-reconstructed knee on kinematics and ACL graft force: a biomechanical analysis. Am J Sports Med. https://doi.org/10.1177/03635465209761473635 46520976147 
8. Imhoff FB, Mehl J, Comer BJ, Obopilwe E, Cote MP, Feucht MJ et al (2019) Slope-reducing tibial osteotomy decreases ACL-graft forces and anterior tibial translation under axial load. Knee Surg Sports TraumatolArthrosc 27:3381-3389

9. Salmon LJ, Heath E, Akrawi H, Roe JP, Linklater J, Pinczewski LA (2018) 20-year outcomes of anterior cruciate ligament reconstruction with hamstring tendon autograft: the catastrophic effect of age and posterior tibial slope. Am J Sports Med 46:531-543

10. Shelbourne KD, Benner RW, Jones JA, Gray T (2021) Posterior tibial slope in patients undergoing anterior cruciate ligament reconstruction with patellar tendon autograft: analysis of subsequent ACL graft tear or contralateral ACL tear. Am J Sports Med 49:620-625

11. Song GY, Ni QK, Zheng T, Zhang ZJ, Feng H, Zhang H (2020) Slope-reducing tibial osteotomy combined with primary anterior cruciate ligament reconstruction produces improved knee stability in patients with steep posterior tibial slope, excessive anterior tibial subluxation in extension, and chronic meniscal posterior horn tears. Am J Sports Med 48:3486-3494

12. Sonnery-Cottet B, Mogos S, Thaunat M, Archbold P, Fayard JM, Freychet B et al (2014) Proximal tibial anterior closing wedge osteotomy in repeat revision of anterior cruciate ligament reconstruction. Am J Sports Med 42:1873-1880
13. Weinberg DS, Williamson DF, Gebhart JJ, Knapik DM, Voos JE (2017) Differences in medial and lateral posterior tibial slope: an osteological review of 1090 tibiae comparing age, sex, and race. Am J Sports Med 45:106-113

14. Winkler PW, Wagala NN, Hughes JD, Lesniak BP, Musahl V (2021) A high tibial slope, allograft use, and poor patient-reported outcome scores are associated with multiple ACL graft failures. Knee Surg Sports TraumatolArthrosc. https://doi.org/10.1007/ s00167-021-06460-8

15. Yamaguchi KT, Cheung EC, Markolf KL, Boguszewski DV, Mathew J, Lama CJ et al (2018) Effects of anterior closing wedge tibial osteotomy on anterior cruciate ligament force and knee kinematics. Am J Sports Med 46:370-377

16. Yoon KH, Park SY, Park JY, Kim EJ, Kim SJ, Kwon YB et al (2020) Influence of posterior tibial slope on clinical outcomes and survivorship after anterior cruciate ligament reconstruction using hamstring autografts: a minimum of 10-year follow-up. Arthroscopy $36: 2718-2727$

Publisher's Note Springer Nature remains neutral with regard to jurisdictional claims in published maps and institutional affiliations. 